\title{
ASCA Observations of the Eclipsing IP XY Ari
}

Eric M. Schlegel

Smithsonian Astrophysical Observatory, 60 Garden St. Cambridge, MA 02138

Koji Mukai

NASA-GSFC/USRA, Code 662, Greenbelt, MD 20771

M. Ishida

\section{ISAS, Yoshinodai 3-1-1, Sagamihara, Kanagawa 229, Japan}

Abstract. We describe the ASCA observation of the eclipsing intermediate polar (IP) XY Ari $(=\mathrm{H} 0253+19)$. This system was first discussed by Patterson \& Halpern (1990, ApJ, 361, 173) as a possible IP based upon the HEAO-1 data. This system is impossible to study in the optical or ultraviolet because of the $12 \mathrm{mag}$ of optical extinction associated with the molecular cloud Lynds $1457=$ MBM 12 . The system has an orbital period of 0.2526 day and a pulse period of $208 \mathrm{sec}$. Our preliminary analysis of the $A S C A$ data shows that the eclipse is nearly total (the precision of the background subtraction sets the limit). The eclipse ingress and egress occur very quickly, although we have not yet derived accurate numbers. There are sufficient photons that we can perform crude phaseresolved spectroscopy. The resulting spectra, each covering about $1 / 4$ of the orbit, show that the Fe $\mathrm{K} \alpha$ line is not constant as a function of phase. A sample spectrum is reproduced below. We expect to submit a paper describing the results to the ApJ before the end of 1996 .

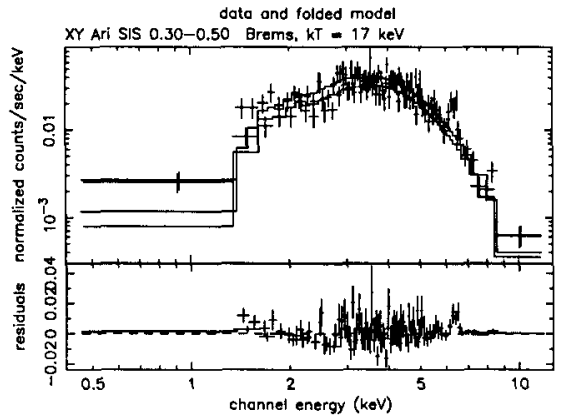

Figure 1. ASCA SIS spectrum of XY Ari, phases $0.30-0.50$ 